\title{
EBSD Characterization of Residual Plastic Strain Across Alloy 182 Weld Joints
}

\author{
M. A. Othon and M. M. Morra \\ GE Global Research Center, 1 Research Circle, Niskayuna, NY 12309
}

Alloy 182 is a Ni-based weld filler metal that is used in applications requiring high strength at intermediate temperatures. Recent studies have shown that in service stress-corrosion cracking (SCC) in the base metal adjacent to the weld heat affected zone (HAZ) can be related to residual plastic strains present after welding [1]. EBSD has been shown to be an effective tool for measuring both microstructure (grain and phase distribution, orientation) and plastic strain.

In this study an alloy 182 weldment made by shielded metal arc welding two alloy 600 plates together was mapped for plastic strain distributions using EBSD. Data collection was performed using HKL s Channel 5 software package. Measurement locations were dictated by the crack paths in two compact tension (CT) specimens that will be extracted from the weldment at the conclusion of this study. To map the residual plastic strain field, three locations were measured horizontally across the weld and HAZ: $7.7 \mathrm{~mm}, 20 \mathrm{~mm}$, and $32.3 \mathrm{~mm}$ from the face of each CT specimen. To map the probable crack paths during SCC growth rate testing, additional measurements were made longitudinal to the machined notches in the CT specimens. (Figure 1.)

Previous studies on welds focused on mapping plastic strain as a function of distance from the highly deformed weld HAZ to the relatively relaxed base material in stainless steels [2]. For the current study, similar regions of the weldment were measured. The primary emphasis, however, was on the plastic strain within the alloy 182 weld metal. Quantification of all measurements were made by calculating the average intragrain misorientation (AMIS) for each line, then plotting the data as a function of distance from one side of the weld to the other. From calibration samples, AMIS data is then converted to plastic strain percentages and plotted [3]. The results from all EBSD measurement locations are shown in Figure 2, rendered using Golden Software s Surfer (v. 8) which interpolates between data sets then plots the data on independent XYZ scales. As can be seen, the maximum plastic strain occurs in the heat-affected zones nearest the weld root, also the region most prone to SCC. Vertical EBSD scans of the weld metal (not shown here but to be presented) show that plastic strain decreases with increasing vertical distance from the weld root.

Another important aspect of this study was to visually benchmark the crystallographic and grain boundary type information for the weld metal along the CT crack front. For the bottom CT specimen, the weld encompasses $\sim 16.4 \mathrm{~mm}$ of material. By using the DAPP (dynamic adjustment of projection parameters) feature in the acquisition software, the entire length of the weld was measured. DAPP measures a series of low magnification beam scanned maps, then stitches the maps together for one continuous image. (Figure 3.) Upon the completion of SCC growth rate testing, the samples will be mapped again at the same locations to determine the effect of dendrite orientation on the crack path.

\section{References}

[1] T.M. Angeliu, Microstructural Characterization of L-Grade Stainless Steels Relative to the IGSCC Behavior in BWR Environments , Corrosion/01, paper 1121. 
[2] Angeliu, T. M., P. L. Andresen, et al. (1999). Intergranular Stress Corrosion Cracking of Unsensitized Stainless Steels in BWR Environments. $9^{\text {th }}$ Int Sympon Env. Deg. Of Mtls. In Nuclear Power Systems TMS

[3] J.A. Sutliff, An Investigation of Plastic Strain in Copper by Automated-EBSP , Microscopy and Micronanalysis, Vol 5. Supp. 21999 p. 236, (Proceedings: Microscopy \& Microanalysis 99)

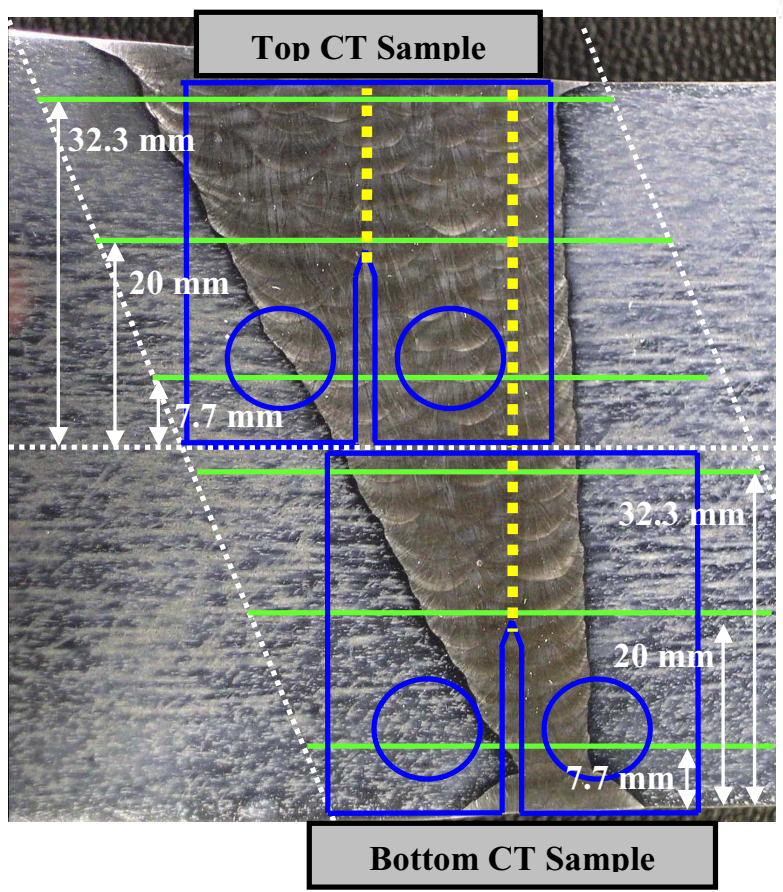

Fig. 1. Light micrograph of weld showing relative positions of horizontal analysis lines (solid green), vertical analysis lines relative to the crack propagation path (dotted yellow), and compact tension specimens (blue outline).

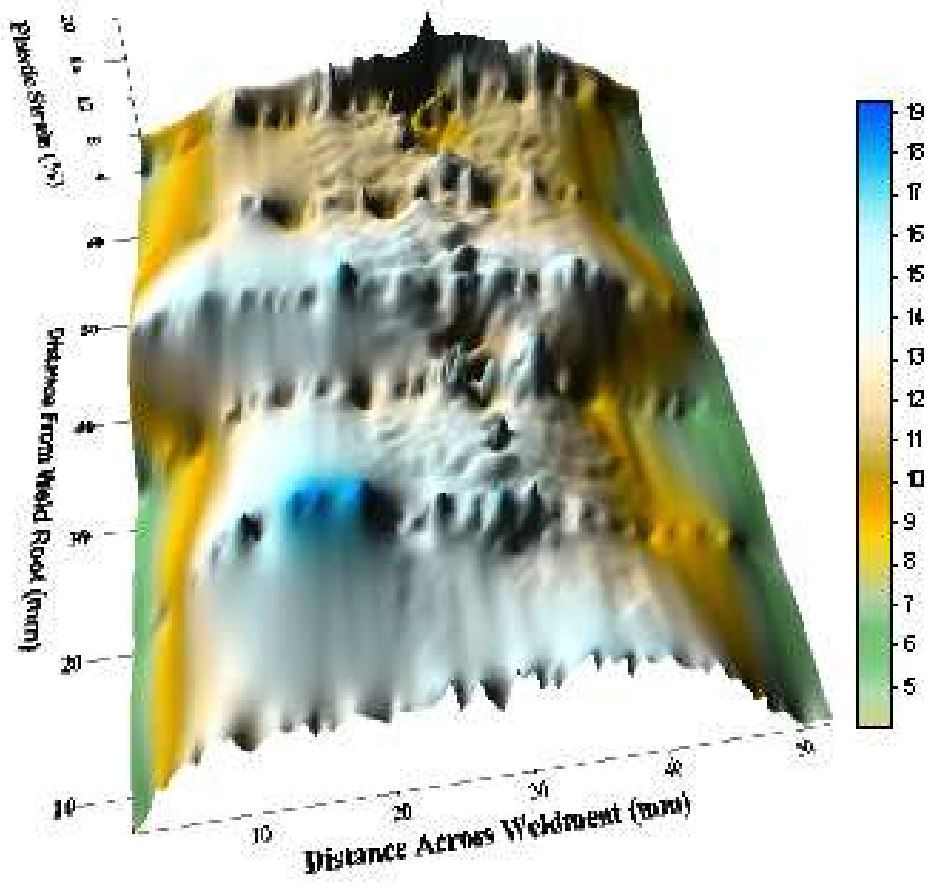

Fig. 2. Plastic strain profile across Alloy 182 weldment. The measured residual plastic strain in the weld metal was found to range from 10 to $18 \%$.

Area of measurement
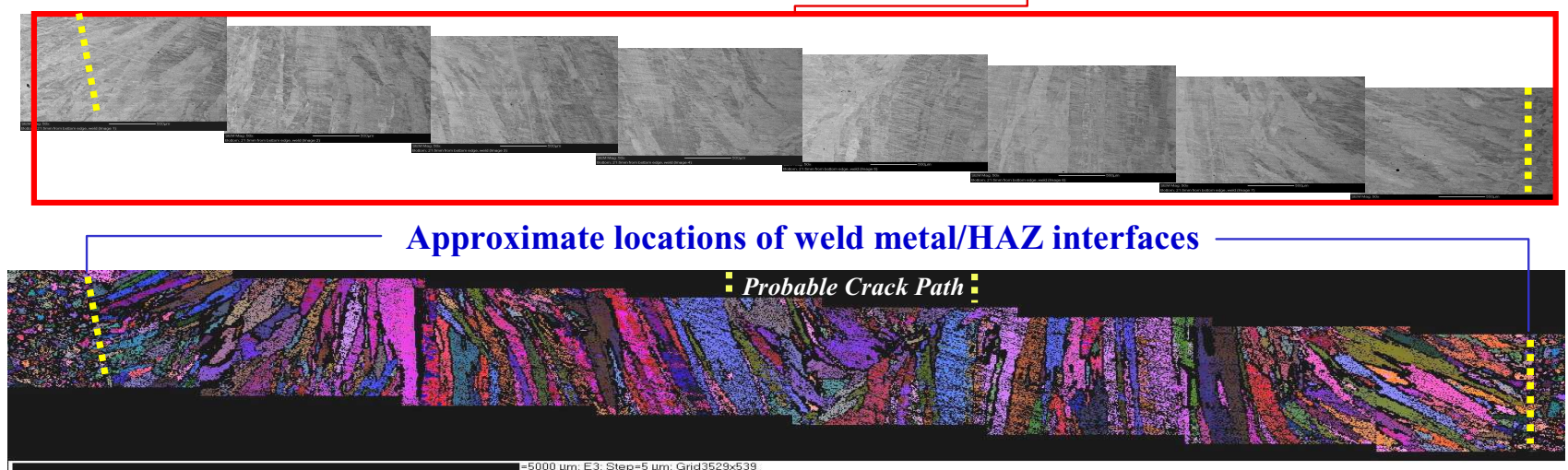

Fig. 3. Euler Map of the entire bottom $20 \mathrm{~mm}$ line obtained by the DAPP technique. Total length of measurement area is $\sim 16.4 \mathrm{~mm}$. 